\title{
Sleep apnoea and post-stroke vascular events: is it only due to poor continuous positive airway pressure use?
}

\section{To the Editors:}

I read the recent European Respiratory Journal article by MARTínEZGARCíA et al. [1] with keen interest. The authors analysed 166 patients with recent ischaemic stroke and followed them for 7 yrs to study the impact of obstructive sleep apnoea (OSA) on new cardiovascular events. According to their results, 31 patients did not have OSA, 39 patients had mild-to-moderate OSA and 96 patients had moderate-to-severe OSA. Continuous positive airway pressure (CPAP) was initiated 2 months after an acute cerebrovascular event in patients with moderate-to-severe disease only. However, 68 out of 96 participants in the severe OSA group did not tolerate CPAP. It is noteworthy to emphasise that noncompliant patients with moderate-to-severe OSA were older, and had a higher prevalence of arterial hypertension, previous cerebrovascular disease, atrial fibrillation (AFib), oral anticoagulant use, antihypertensive medication use and diabetes mellitus, but except for age, these variables did not reach statistical significance. After regression analysis adjusted for potential confounders, such as age, sex, stroke severity, previous cerebrovascular disease, AFib, arterial hypertension, previous ischaemic heart disease, hypercholesterolaemia, fibrinogen levels, carotid artery disease, smoking status and body mass index, the authors found that untreated OSA independently increased the nonfatal cardiovascular risk (predominantly cerebrovascular disease) 2.87 times $(p=0.033)$. Overall, this study solidifies current knowledge (the first study with such long follow-up) that OSA is a strong cardiovascular risk factor and the speculations about possible protective effects on decreased vascular morbidity and mortality among elderly patients with OSA are of less value, at least for stroke [2]. Another key issue highlighted by the study is that clinical questionnaires for excessive daytime sleepiness (EDS) may have decreased sensitivity in stroke patients or these patients may not have EDS at all. I would like to thank and congratulate the authors on this exceptionally important data. However, some issues need to be mentioned.

First, it is important to note that there was no information about which hypotensive medications were used by participants, since different classes have distinct effects on stroke prevention [3]. Secondly, no information is present on whether patients were receiving statins after stroke, which is of significant relevance for stroke prevention too [4]. Thirdly, no information about change of cardiovascular risk factors during the follow-up period is available. Fourthly, as mentioned by the authors, CPAP-noncompliant patients had a higher prevalence of some cardiovascular risk factors, pointing to possible problems with general treatment compliance, and thus a more advanced vascular disease. Overall, OSA seems to be a strong, independent risk factor for diabetes mellitus, arterial hypertension and cerebrovascular disease; however, these diseases have complex pathogenesis and definitively require multitargeted therapy. Therefore, poor control of comorbid conditions during the follow-up period may detrimentally affect the excessive cardiovascular risk seen in OSA.

Finally, the study has showed that patients with recent stroke have particularly poor CPAP adherence, which can have detrimental consequences for their health. Approximately 13-14 nonfatal cardiovascular events could theoretically be prevented if all the participants used their CPAP machines. This is a very important issue, which definitively requires further research on how we can improve OSA treatment adherence. Recent work in a small number of patients with acute stroke has pointed out that transnasal insufflation via a nasal cannula has good compliance and beneficial effects on OSA severity [5]. However, more research is urgently needed to improve patients' compliance to the treatment, which can have a positive impact on their prognosis.

\begin{abstract}
Aibek E. Mirrakhimov ${ }^{*, \#}$
*Kyrgyz State Medical Academy named after I.K. Akhunbaev, and "National Centre of Cardiology and Internal Medicine named after M. Mirrakhimov, Bishkek, Kyrgyzstan.
\end{abstract}

Correspondence: A. E. Mirrakhimov, National Centre of Cardiology and Internal Medicine named after M. Mirrakhimov, T. Moldo 3 Street, Bishkek, 720040, Kyrgyzstan. E-mail: amirrakhimov1@gmail.com

Statement of Interest: None declared.

\section{REFERENCES}

1 Martínez-García MA, Campos-Rodríguez F, Soler-Cataluña JJ, et al. Increased incidence of nonfatal cardiovascular events in stroke patients with sleep apnoea: effect of CPAP treatment. Eur Respir J 2012; 39: 906-912.

2 Lavie L, Lavie P. Ischemic preconditioning as a possible explanation for the age decline relative mortality in sleep apnea. Med Hypotheses 2006; 66: 1069-1073.

3 Armario P, de la Sierra A. Antihypertensive treatment and stroke prevention: are angiotensin receptor blockers superior to other antihypertensive agents? Ther Adv Cardiovasc Dis 2009; 3: 197-204.

4 Flint AC, Kamel H, Navi BB, et al. Statin use during ischemic stroke hospitalization is strongly associated with improved poststroke survival. Stroke 2012; 43: 147-154.

5 Haba-Rubio J, Andries D, Rey V, et al. Effect of transnasal insufflation on sleep disordered breathing in acute stroke: a preliminary study. Sleep Breath 2011; [Epub ahead of print DOI: 10.1007/s11325-0110572-3].

DOI: 10.1183/09031936.00054412 